\title{
January 2018 Imaging Case of the Month
}

\author{
Michael B. Gotway, MD \\ Department of Radiology \\ Mayo Clinic Arizona \\ Scottsdale, AZ USA
}

Clinical History: An 81-year-old woman with little significant past medical history complained of a dry cough for the previous 1.5 years, but without hemoptysis or shortness of breath. The patient's past medical history was remarkable only for hypothyroidism, for which she was taking levothyroxine. She smoked for 1 year only, at age 19. Her past surgical history was negative and she denied any alcohol use. Her only other medications included vitamin D3 supplementation and over-the-counter cough medicine.

Physical examination was remarkable only for coarse, left-greater-than-right basal rales. The patient's oxygen saturation was $98 \%$ on room air. The patient's vital signs were within normal limits and she was afebrile.

Laboratory evaluation showed a normal complete blood count, electrolyte panel, and liver function tests. Frontal chest radiography (Figure 1) was performed.

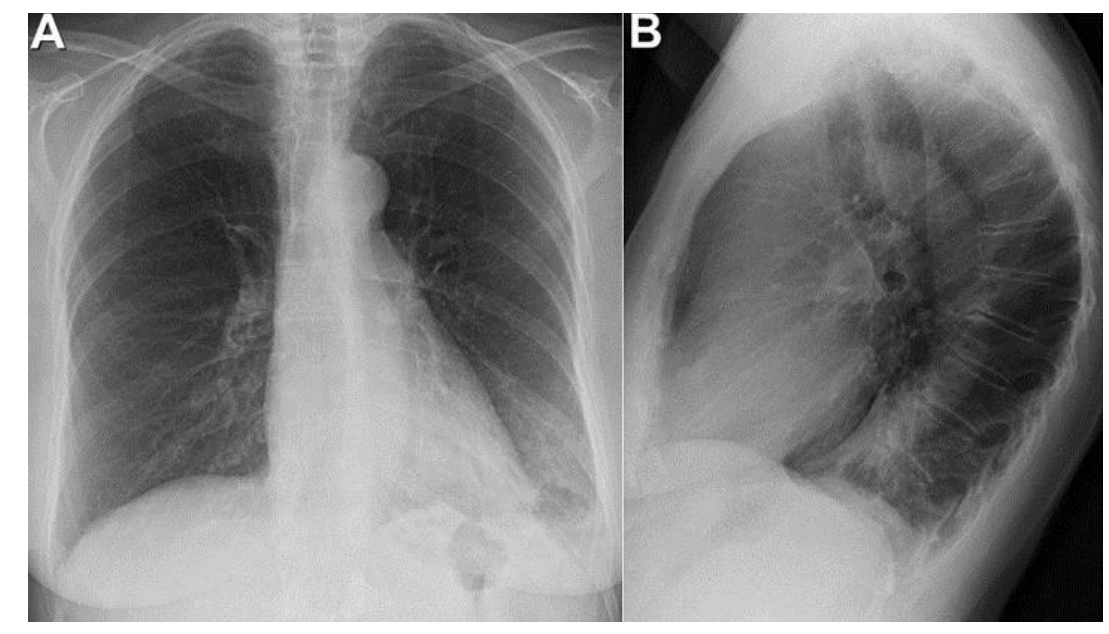

Figure 1. Frontal $(A)$ and lateral $(B)$ chest radiography.

Which of the following represents the most accurate assessment of the chest radiographic findings?

1. Chest radiography shows basilar fibrosis

2. Chest radiography shows left lower lobe consolidation

3. Chest radiography shows mediastinal and peribronchial lymphadenopathy.

4. Chest radiography shows multiple small nodules

5. Chest radiography shows normal findings 


\section{Correct! \\ 2. Chest radiography shows left lower lobe consolidation}

The chest radiograph shows normal hilar and mediastinal contours- no lymphadenopathy is evident. There is no basal reticulation to suggest fibrotic lung disease, and the lung volumes appear normal. No discrete nodules are present. There is focal left basal opacity, best seen on the lateral chest radiograph, consistent with left lobe consolidation associated with some volume loss (Figure 2).

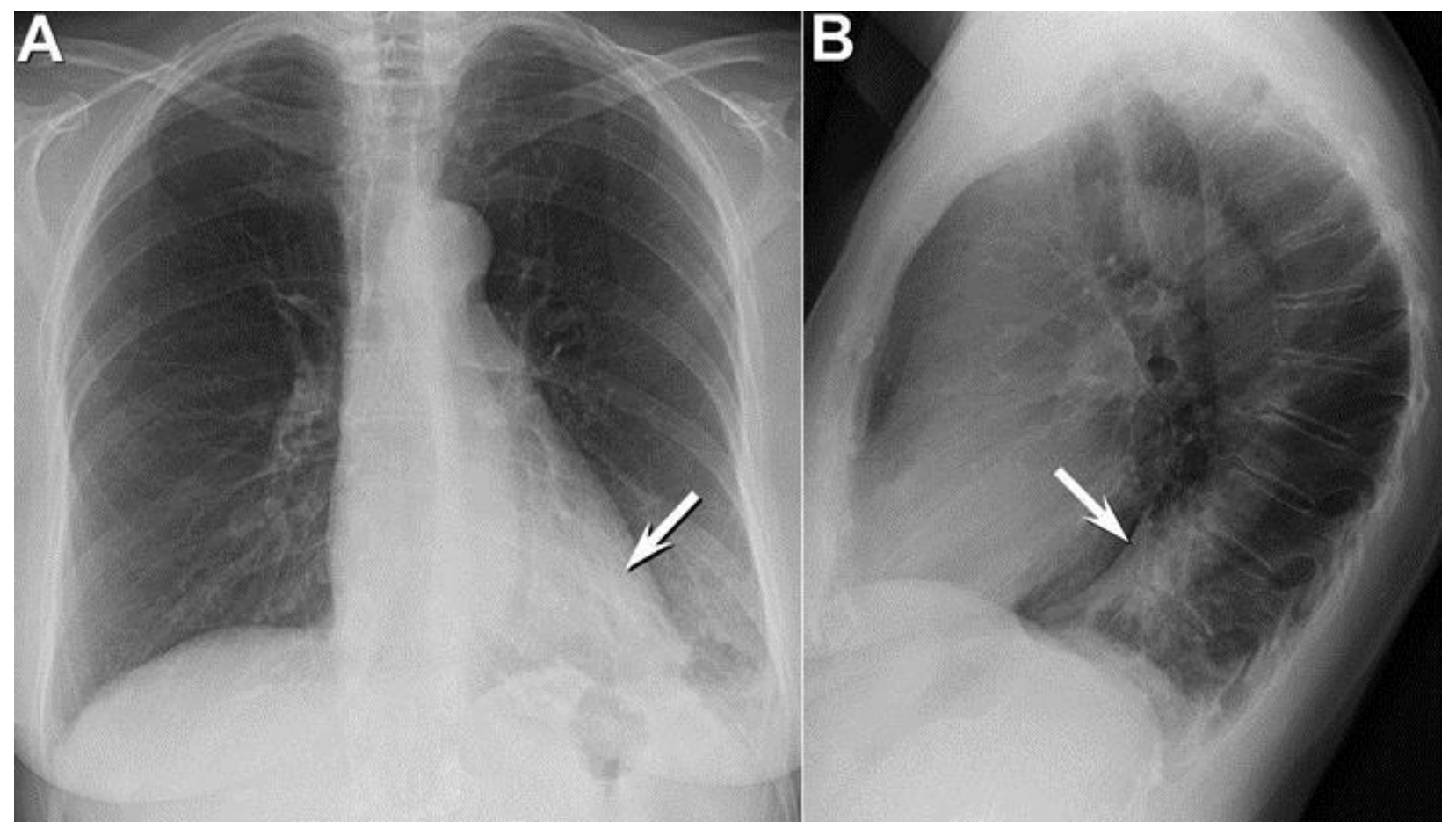

Figure 2. Frontal $(A)$ and lateral $(B)$ chest radiography shows left lower lobe consolidation (arrows).

The patient was not febrile.

Which of the following represents the most appropriate management step for the evaluation of this patient?

1. Check titers for possible fungal infection, treat presumptively with broadspectrum antibiotics for presumed pneumonia

2. Obtain ${ }^{18} \mathrm{FDG}$-PET scan

3. Obtain abdominal CT

4. Obtain surgical lung biopsy

5. Perform pleuroscopy 


\section{Correct! \\ 1. Check titers for possible fungal infection, treat presumptively with broad- spectrum antibiotics for presumed pneumonia}

Among the choices listed, treating with broad-spectrum antibiotics for presumed pneumonia, and checking for titers suggesting fungal infection, particularly coccidioidomycosis (given that this infection is responsible for a significant proportion of community-acquired pneumonias) is appropriate. The chest radiographic findings, while not specific for pneumonia alone, are certainly consistent with that diagnosis. The lack of fever and the normal white blood cell count are unusual findings for pulmonary infection, but that diagnosis remains a consideration, and, "common things being common," the diagnosis of community-acquired pneumonia remains a strong possibility. The patient has no pleural effusion and therefore pleuroscopy is not an appropriate choice. Surgical lung biopsy is needlessly invasive at this point. ${ }^{18} \mathrm{FDG}-\mathrm{PET}$ probably would not provide management-altering results- active tracer utilization within the area of left lower lobe consolidation would be expected in the setting of pneumonia. The lack of tracer utilization within the left lower lobe abnormality would be a peculiar result that would be difficult to interpret. Most infections would be expected to result in tracer utilization, but the lack of an intense inflammatory response could result in false negative findings at ${ }^{18} \mathrm{FDG}-\mathrm{PET}$.

The patient' was treated with broad-spectrum antibiotics for several weeks and the patient's cough improved somewhat. The patient remained afebrile. Testing for various infectious agents was pending. Frontal and lateral chest radiography (Figure 3) was repeated.

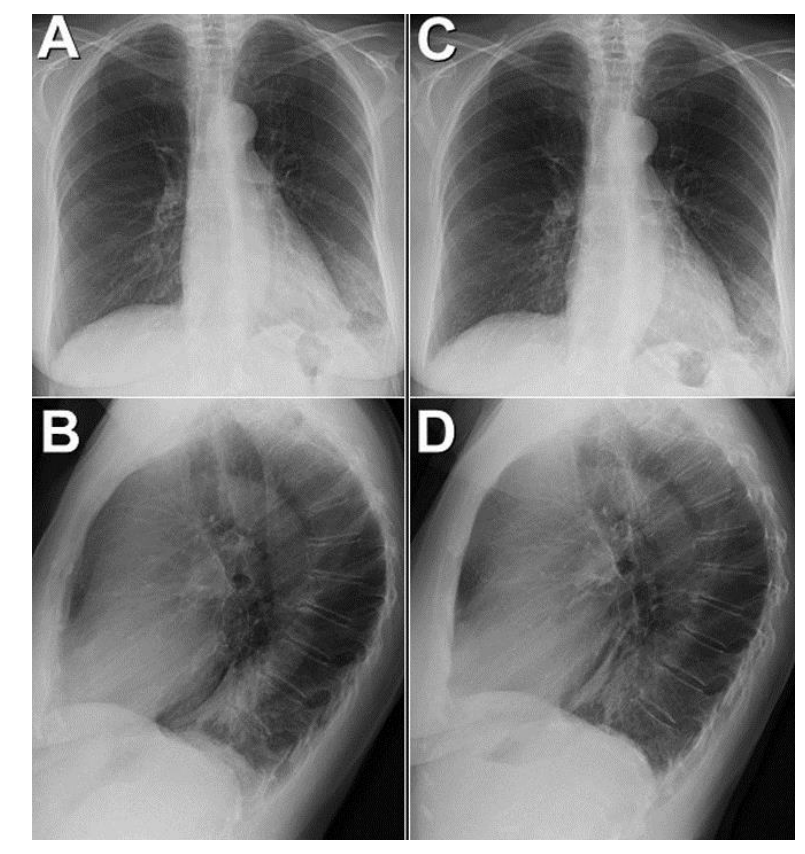

Figure 3. Repeat frontal (A) and lateral (B) chest radiography. Chest radiograph from 2 weeks earlier ( $C$ and $D)$ is shown for comparison. 
Which of the following represents the most accurate assessment of the repeat chest radiographic findings?

1. Repeat chest radiography shows development of pleural effusion

2. Repeat chest radiography shows improvement in the left lower lobe opacity

3. Repeat chest radiography shows interval development of peribronchial and mediastinal lymph node enlargement

4. Repeat chest radiography shows new multifocal opacities bilaterally

5. Repeat chest radiography shows worsening of the left lower lobe opacity 


\section{Correct!}

\section{Repeat chest radiography shows improvement in the left lower lobe opacity}

The repeat chest radiograph shows some improvement in the focal left lower lobe opacity compared to the presentation chest radiograph, although some abnormality remains. There is no evidence of progressive abnormality- the left lower lobe opacity is not clearly worsened, there is no pleural effusion, no new opacities are present, and there is no evidence of new peribronchial or mediastinal lymphadenopathy.

The patient's coccioidomycosis testing was unrevealing. While her cough briefly improved somewhat after the initiation of broad-spectrum antibiotics, her cough did persist, although she remained afebrile. Frontal and lateral chest radiography (Figure 4) was repeated.

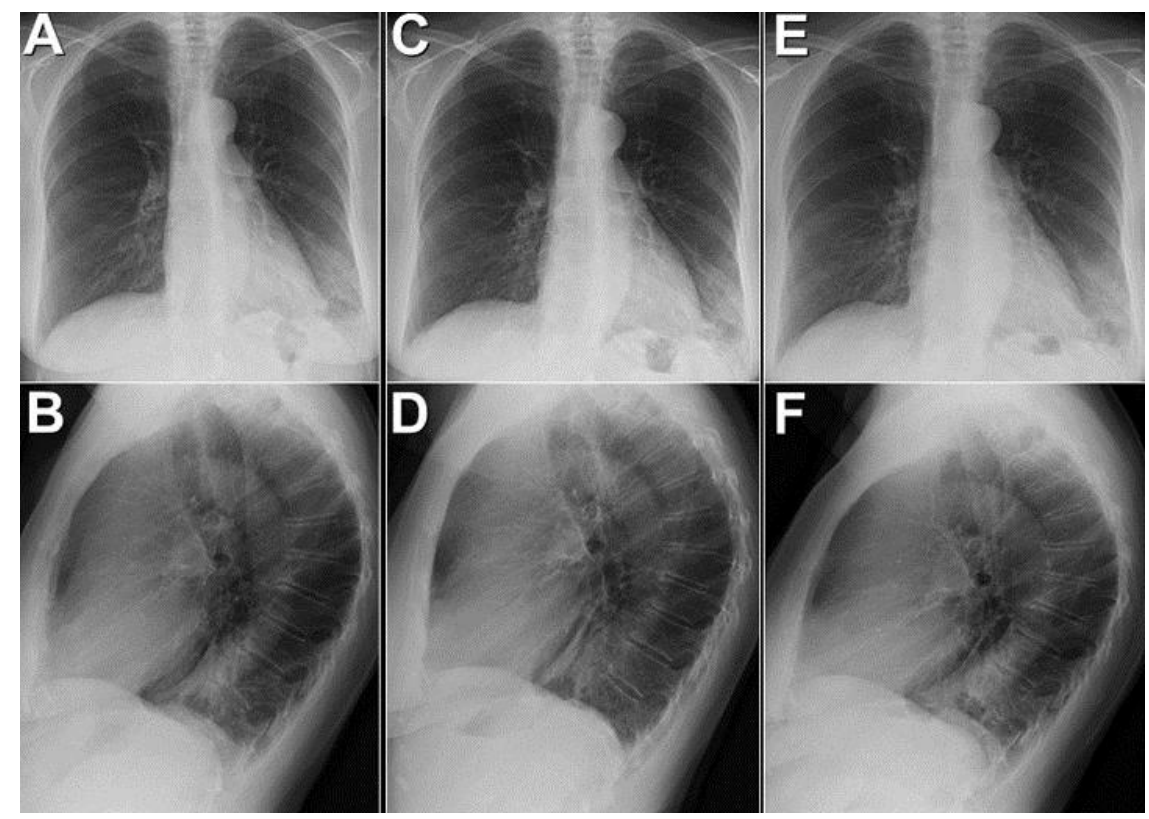

Figure 4. Frontal $(A)$ and lateral $(B)$ chest radiography performed 3 months after initial presentation chest radiography $(E \& F)$ shows that the left lower lobe consolidation is again present, similar to that seen at initial presentation. The chest radiography done 2 weeks after the initial radiography $(C \& D)$ is shown for comparison.

Which of the following statements the most accurate assessment of the repeat chest radiographic findings?

1. Repeat chest radiography shows complete clearing of the left lower lobe opacity

2. Repeat chest radiography shows further improvement in the left lower lobe opacity

3. Repeat chest radiography shows interval development of peribronchial and mediastinal lymph node enlargement

4. Repeat chest radiography shows new multifocal opacities bilaterally

5. Repeat chest radiography shows persistence of the left lower lobe opacity 


\section{Correct! \\ 5. Repeat chest radiography shows persistence of the left lower lobe opacity}

The repeat chest radiograph shows that the left lower lobe opacity is still present, similar to the appearance at the presentation chest radiograph (Figure 1) 3 months earlier. The left lower lobe opacity is not clearly worsened since the presentation chest radiograph, but is not improved since that time. However, there is no evidence of new or progressive abnormality- there is no pleural effusion, no new opacities are present, and there is no evidence of new peribronchial or mediastinal lymphadenopathy.

Which of the following represents the most appropriate next step for the management of this patient?

1. ${ }^{68} \mathrm{Ga}-\mathrm{PET}-\mathrm{CT}$ dotatate scan

2. Continue observation

3. Obtain ${ }^{18}$ FDG-PET scan

4. Perform cardiac MRI

5. Perform thoracic CT 


\section{Correct! \\ 5. Perform thoracic CT}

Given the persistence of the left lower lobe opacity, negative testing for infections, and lack of fever and elevated white blood cell count, infection, while not entirely excluded, is increasingly unlikely; therefore, continued observation is not appropriate. Cardiac MRI would not be indicated for this patient. ${ }^{68} \mathrm{Ga}-\mathrm{PET}$ - CT dotatate scanning is typically used to stage neuroendocrine cell malignancies and would not clearly be of benefit for this patient at this point. 18FDG-PET scanning may be of benefit, but is usually most rewarding when interpreted in the context of dedicated anatomic imaging, typically thoracic CT; therefore, thoracic CT is the best answer among the choices offered.

The patient underwent unenhanced thoracic CT (Figure 5).

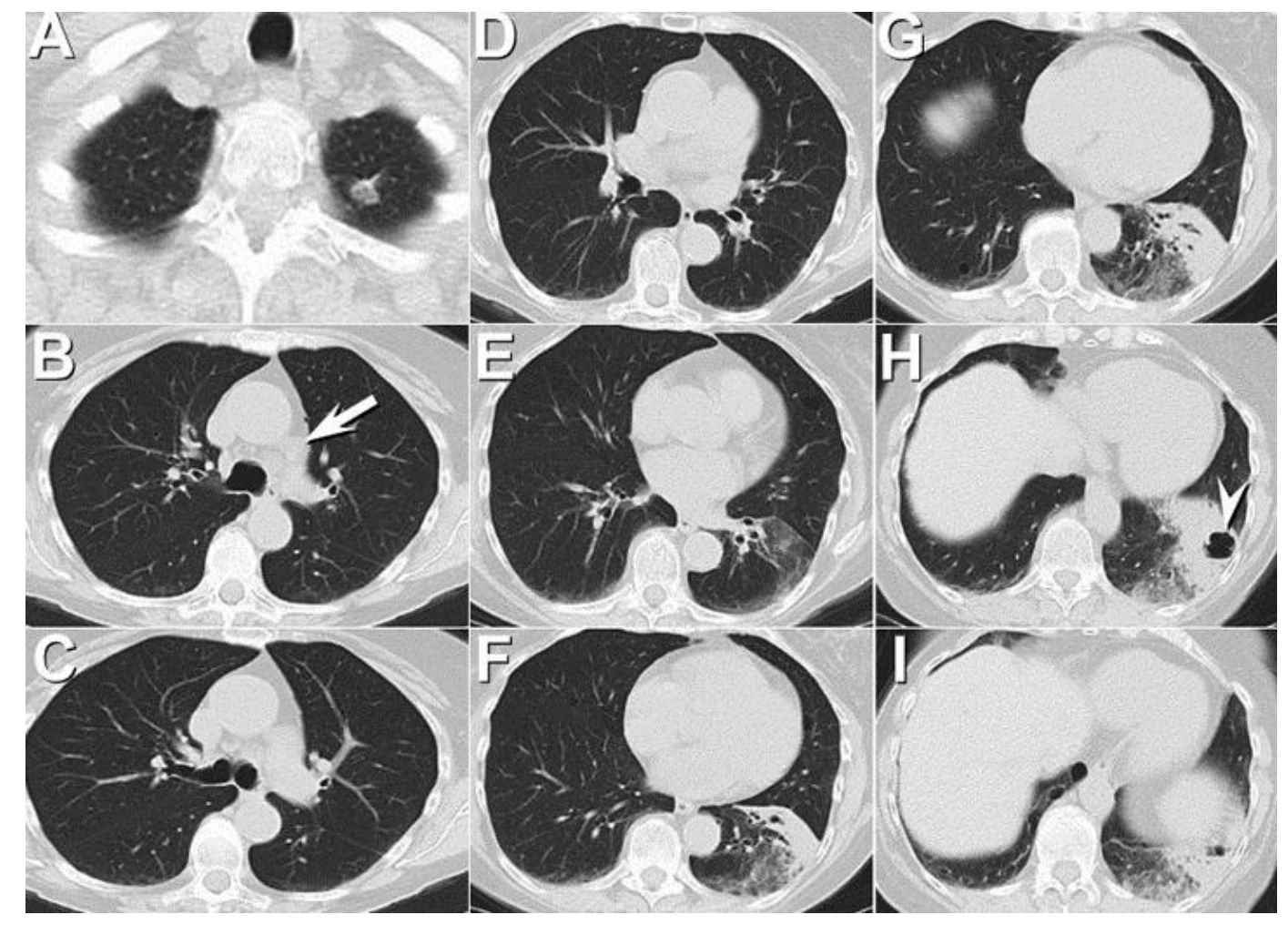

Figure 5. Representative images from unenhanced thoracic CT, performed close to 6 months after initial presentation.

Which of the following represents the most accurate assessment of the thoracic CT findings?

1. The thoracic CT shows a left diaphragmatic hernia

2. The thoracic CT shows a left lower lobe mass

3. The thoracic CT shows focal left lower lobe bronchial obstruction

4. The thoracic CT shows left lower lobe consolidation with central cavitation

5. The thoracic CT shows new, loculated left base pleural effusion 


\section{Correct!}

\section{The thoracic CT shows left lower lobe consolidation with central cavitation}

The thoracic CT shows left lower lobe consolidation and ground-glass opacity, with some volume loss evidenced by posterior retraction of the left major fissure, but the left lower lobe airways are clearly patent- no endobronchial obstruction is present.

Cavitation is present within the densest area of consolidation within the left lower lobe opacity. While diaphragmatic hernias can occasionally simulate the presence of a left base pneumonia or even a left base mass, no significant diaphragmatic hernia is present. No pleural effusion, loculated or otherwise, is seen. There is focal consolidation in the left base [note the air bronchograms], but no discrete mass is present.

The patient's cough persisted, relatively unchanged.

Given the foregoing information, which of the following represents the most

appropriate next step for the management of this patient?

1. Obtain ${ }^{18}$ FDG-PET scan

2. Obtain video-assisted thoracoscopic surgery

3. Perform endoscopic ultrasound

4. Perform percutaneous transthoracic needle biopsy of the left lower lobe process

5. Perform repeat thoracic CT using a high-resolution CT protocol 


\section{Correct! \\ 1. Obtain ${ }^{18}$ FDG-PET scan or 4 . Perform percutaneous transthoracic needle biopsy of the left lower lobe process}

Among the choices listed, obtaining ${ }^{18}$ FDG-PET scan is most appropriate. Repeating the thoracic CT as a high-resolution CT [HRCT] protocol would be of little benefit. HRCT is a "sampling" technique, typically used for the assessment of diffuse lung disease, not focal lung opacities, as seen in this patient. Video-assisted thoracoscopic surgery [VATS] could be of benefit in this patient, but is probably overly-invasive- other less invasive and less expensive options for establishing a diagnosis for this patient exist. Endoscopic ultrasound, however, is not one of them- this procedure may be of diagnostic utility if the targeted lesion is in close proximity to the esophagus or stomach, which is not the case for this patient. Percutaneous transthoracic fine needle biopsy could potentially establish a diagnosis for this patient and is not an inappropriate choice, but among the invasive procedures that could be used to assess this patient, bronchoscopy- which was not offered as one of the choices listed- would probably be the best procedure.

${ }^{18}$ FDG-PET scan (Figure 6) was performed.

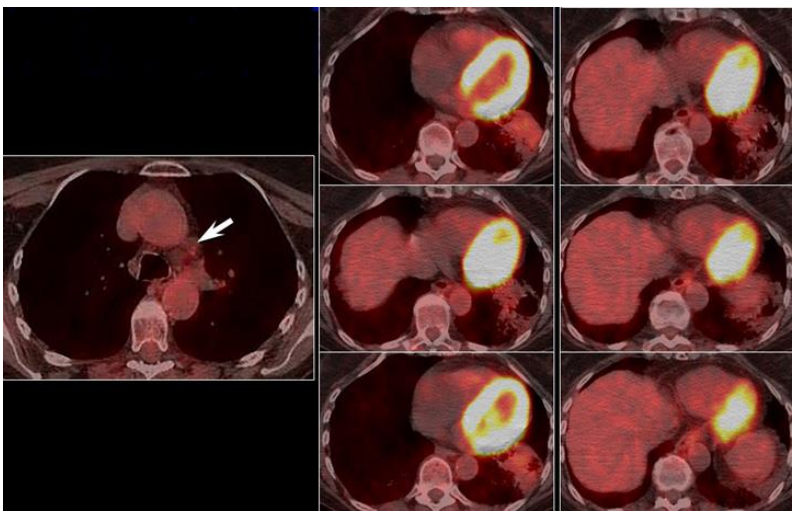

Figure 6. ${ }^{18} \mathrm{FDG}$-PET shows no significant tracer accumulation within the region of left lower lobe consolidation. The borderline enlarged subaortic lymph node (arrow) seen at thoracic CT shows no significant tracer accumulation.

This study showed no clearly significant tracer utilization within the left lower lobe cavitary opacity, the left apical nodule, or mediastinal lymph nodes.

Which of the following represents the most appropriate next step for the management of this patient?

1. Obtain video-assisted thoracoscopic surgery

2. Perform a Chamberlain procedure

3. Perform bronchoscopy

4. Perform open surgical biopsy

5. Perform percutaneous transthoracic needle biopsy of the left lower lobe process 


\section{Correct!}

\section{Obtain video-assisted thoracoscopic surgery; or 3. Perform bronchoscopy; or 5. Perform percutaneous transthoracic needle biopsy of the left lower lobe process}

While open surgical lung biopsy is needlessly invasive, performing bronchoscopy, video-assisted surgical lung biopsy, or percutaneous transthoracic needle biopsy are all relatively appropriate choices for obtaining tissue in this patient, although bronchoscopy is probably the favored choice. Pleuroscopy is useful for assessment of pleural effusions or pleural masses, but neither are present in this patient. The Chamberlain procedure can be useful for sampling left para-aortic, anterior mediastinal, prevascular, and subaortic lesions, including lymph nodes in these stations, but the subaortic lymph node in this patient was only borderline-enlarged at CT and not metabolically active at ${ }^{18} \mathrm{FDG}-\mathrm{PET}$, and probably is not an appropriate target for obtaining a tissue diagnosis.

The patient's cough persisted relatively unchanged. She underwent repeat thoracic CT (Figure 7).

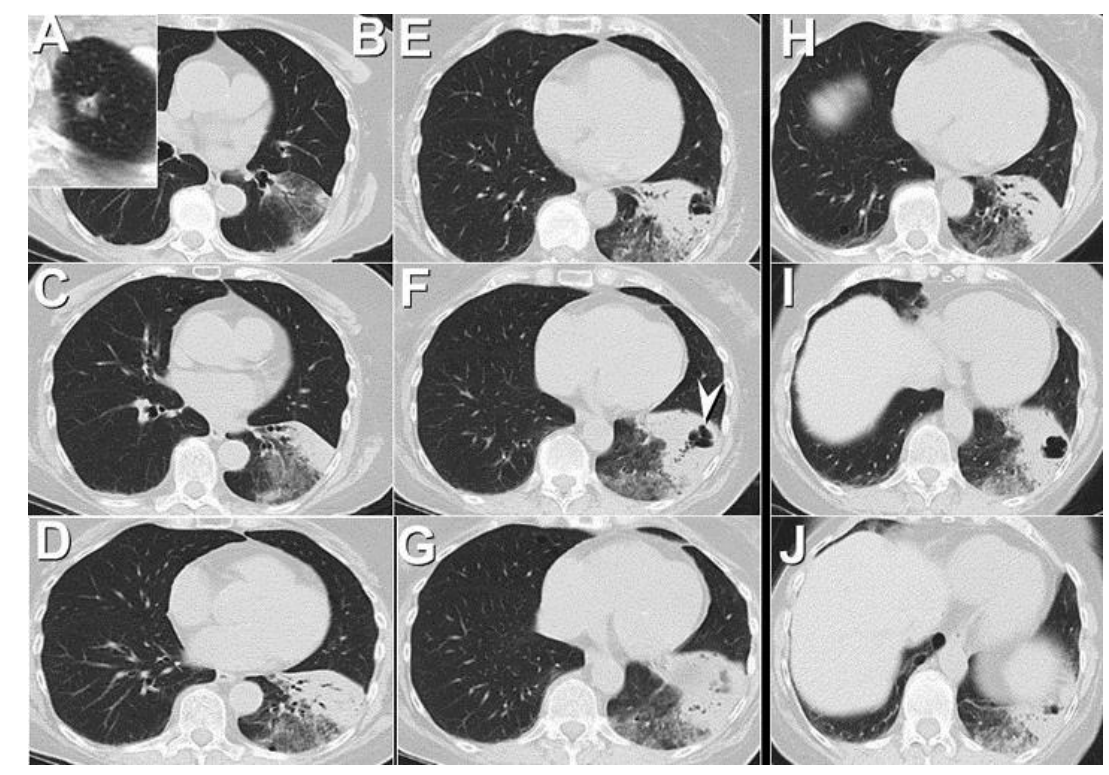

Figure 7. Representative images from an unenhanced thoracic CT performed nearly 12 months after initial presentation. Panels $\mathrm{H}-\mathrm{J}$ are images from the previous thoracic CT (Figure 5), performed nearly 6 months earlier.

Which of the following represents the most accurate assessment of the thoracic CT findings?

1. The thoracic CT shows development of new right-sided opacities

2. The thoracic CT shows enlargement of the left apical nodule

3. The thoracic CT shows marked progression in the left base process

4. The thoracic CT shows new left base pleural effusion

5. The thoracic CT shows relatively little change in the left lower lobe process 


\section{Correct!}

\section{The thoracic CT shows marked progression in the left base process}

The thoracic CT, performed nearly one year after initial presentation, shows relatively little interval change in the left lower lobe ground-glass opacity, consolidation, and cavitation. No new lymph node enlargement in either the peribronchial or mediastinal regions is noted, no new lung opacities on either side are seen, and no pleural abnormality has developed. The left lower lobe airways remain patent. The left apical nodule remains stable. The left base opacity, however, has not regressed.

After the repeat chest CT showed no regression on the left base process, percutaneous transthoracic needle biopsy of the solid-appearing portion of the left lower lobe process was performed.

Which of the following represents an appropriate differential diagnostic consideration for a cavitary pulmonary lesion?

1. Non-Hodgkin lymphoma

2. Pneumonia

3. Primary pulmonary adenocarcinoma

4. All of the above

5. None of the above 


\section{Correct! \\ 4. All of the above}

The differential diagnosis for chronic cavitary consolidation should include neoplasia, particularly primary pulmonary malignancies, such as adenocarcinoma, but also lymphoma. Infection remains with the differential diagnosis, however. While coccidioidomycosis is less likely given the negative testing, negative serology does not necessarily exclude this diagnosis. Furthermore, other indolent infections, including other endemic fungi, or even cryptococcosis, as well as nocardiosis and actinomycosis, could present in this manner.

The patient's percutaneous transthoracic needle biopsy showed mucinous adenocarcinoma with tumor markers consistent with lung origin.

Diagnosis: Mucinous adenocarcinoma presenting as persistent left lower lobe consolidation

\section{References}

1. Sung YM, Lee KS, Kim BT, Han J, Lee EJ. Lobar mucinous bronchioloalveolar carcinoma of the lung showing negative FDG uptake on integrated PET/CT. Eur Radiol. 2005;15(10):2075-8. [CrossRef] [PubMed]

2. Lee KS, Kim Y, Han J, Ko EJ, Park CK, Primack SL. Bronchioloalveolar carcinoma: clinical, histopathologic, and radiologic findings. Radiographics. 1997;17(6):1345-57. [CrossRef] [PubMed]

3. Austin JH, Garg K, Aberle D, Yankelevitz D, Kuriyama K, Lee HJ, Brambilla E, Travis WD. Radiologic implications of the 2011 classification of adenocarcinoma of the lung. Radiology. 2013;266(1):62-71. [CrossRef] [PubMed]

4. Travis WD, Brambilla E, Noguchi M, et al. International association for the study of lung cancer/American thoracic society/European respiratory society international multidisciplinary classification of lung adenocarcinoma. J Thorac Oncol. 2011;6(2):244-85. [CrossRef] [PubMed] 